\title{
CÂNCER DE COTO GÁSTRICO EM RATOS: PAPEL DA ASSOCIAÇÃO DE HELICOBACTER PYLORI E REFLUXO ÊNTERO- GÁSTRICOO
}

\author{
GASTRIC STUMP CANCER IN RATS: ROLE OF THE ASSOCIATION BETWEEN \\ HELICOBACTER PYLORI AND ENTERO-GASTRIC REFLUX
}

\author{
Eduardo Linhares Riello de Mello, TCBC-RJ ${ }^{1}$ \\ Roberto Alfonso Arcuri ${ }^{2}$ \\ Guilherme Pinto Bravo Neto, TCBC $^{3}$ \\ José Humberto Simões, TCBC-RJ ${ }^{4}$
}

\begin{abstract}
RESUMO: Objetivos: Avaliar a relação entre o refluxo êntero-gástrico e Helicobacter pylori na gênese de neoplasias epiteliais e seus precursores, no rato submetido a gastrectomia subtotal com reconstrução a Billroth II. Método: Foram operados 20 ratos machos da raça Wistar, divididos igualmente em dois grupos: um grupo controle submetido à laparotomia exploradora e outro submetido à gastrectomia subtotal a BII. Após nove meses de operados, os animais foram mortos e seus estômagos estudados quanto à quantificação de $H$. pylori por glândula gástrica e à alterações epiteliais neoplásicas ou pré-neoplásicas. Resultados: Os animais gastrectomizados tiveram percentual maior de colonização por $H$. pylori, assim como de alterações epiteliais que variaram do aumento do número de mitoses ao carcinoma invasor. Conclusão: O comprometimento glandular por H. pylori aumenta com o refluxo êntero-gástrico proporcionado pela gastectomia parcial, e está associado ao desenvolvimento de adenocarcinoma de coto gástrico em ratos.
\end{abstract}

Descritores: Neoplasias gástricas; Gastrectomia; Helicobacter pylori; Ratos Wistar.

\section{INTRODUÇÃO}

Apesar do declínio de sua incidência, o câncer gástrico ainda é comum em algumas regiões. Há cerca de 20 anos, no Brasil, correspondia à segunda neoplasia mais freqüente em homens e à quinta no sexo feminino ${ }^{1}$. Estudo mais recente do Ministério da Saúde, o coloca em segundo lugar quanto à incidência e em terceiro quanto à mortalidade ${ }^{2}$. Sua patogênese continua sendo exaustivamente estudada, mas até o momento, nenhum fator isoladamente, pôde ser implicado na carcinogênese gástrica. A associação de fatores, por outro lado, já se revelou significativamente importante no desenvolvimento des- tes tumores, como a ingestão de nitritos e nitratos, que, degradados pela microbiota gástrica, formam n-nitrosaminas, capazes de induzir carcinogênese através da liberação de radicais livres ${ }^{3,4}$. Outros fatores relacionados são o refluxo êntero-gástrico e, mais recentemente, a presença de infecção por H. pylori ${ }^{5,6}$. Esta bactéria Gram-negativa espiralada está freqüentemente associada à gastrite crônica, e é capaz de levar à gastrite atrófica, uma reconhecida condição de risco para câncer gástrico. A infecção por $H$. pylori tem alta prevalência na população, mas apenas uma pequena parcela desenvolve neoplasia, sugerindo que a bactéria não atua isoladamente.

1. Chefe do Serviço de Cirurgia Abdomino-Pélvica do INCa; Mestre e Doutor em Cirurgia pela Universidade Federal do Rio de Janeiro.

2. Patologista do INCa; Mestre e Doutor em Patologia.

3. Professor Adjunto do Departamento de Cirurgia da Faculdade de Medicina da Universidade Federal do Rio de Janeiro.

4. Médico-Cirurgião e Diretor do HC1 do INCa; Mestre em Cirurgia Abdominal.

Trabalho realizado no Laboratório de Pesquisa Básica do INCa; Tese de Doutorado do Curso de Pós-Graduação em Cirurgia da UFRJ. 
Da mesma forma, a indução do câncer pelo refluxo êntero-gástrico em pacientes submetidos à gastrectomia parcial, é determinada pela elevação do pH gástrico, com aumento da proliferação bacteriana, com conseqüente aumento da produção de n-compostos, que em última análise, podem causar metaplasia, displasia e câncer gástrico.

O objetivo deste trabalho é avaliar o comportamento do $H$. pylori na carcinogênese gástrica, em ratos submetidos à gastrectomia parcial com reconstrução a B II, um modelo experimental sabidamente indutor de refluxo êntero-gástrico.

\section{MÉTODO}

Foram utilizados 20 ratos machos da raça Wistar, com idade média de três meses, peso médio de $300 \mathrm{~g}$ e aclimatados às condições do biotério do Instituto Nacional do Câncer (INCa). Nas 24 horas que antecederam ao experimento os animais tiveram acesso somente à água. Os animais foram divididos em dois grupos:

GRUPO I (controle) - constituído por 10 animais submetidos à laparotomia e manipulação do estômago.

GRUPO II (gastrectomia) - constituído por 10 animais submetidos à gastrectomia parcial com reconstrução a B II.

A técnica anestésica utilizada foi a inalação de éter sulfúrico em cuba fechada, para sedação inicial, e ketamina intra-muscular na face anterior da coxa, na dose de $50 \mathrm{mg} / \mathrm{kg}$. Todos os animais foram operados em condições assépticas e entre às 9 e 13 horas. No pós-operatório permitiu-se a alimentação ad libitum.

A reconstrução do trânsito nos animais do Grupo II foi feita através de gastro-jejunostomia oralis-totalis, com alça jejunal situada $2 \mathrm{~cm}$ abaixo da transição duodeno-jejunal. A anastomose foi realizada com visualização microscópica (microscópio Zeiss com objetiva de 10X), em plano total com chuleio simples de prolene 5-0, agulha cilíndrica de $1,5 \mathrm{~cm}$.

Todos os animais foram mantidos no pós-operatório sob as mesmas condições ambientais. Após nove meses de observação foram mortos por inalação de éter em cuba fechada, e os estômagos retirados e abertos pela grande curvatura, presos a isopor para evitar retração e fixados em formol tamponado. Na preparação para análise histopatológica, realizaram-se cortes nos estômagos íntegros em antro, cor- po e fundo. Nos estômagos operados, os cortes foram feitos a nível da anastomose gastro-jejunal, corpo e fundo. As peças cirúrgicas assim tratadas foram submetidas à quantificação de $H$. pylori, conforme método descrito a seguir, e à análise histopatológica visando a identificar alterações de caráter préneoplásico e neoplásico.

Para o estudo histopatológico da mucosa gástrica foi realizada coloração de hematoxilina-eosina e as alterações avaliadas foram a hiperplasia foveolar e displasia. A hiperplasia foveolar foi caracterizada por alongamento, tortuosidade e hipercelularidade glandular ${ }^{7}$. A definição e classificação das displasias obedeceu aos critérios de Ming ${ }^{8}$, e foram divididas em leve, moderada e intensa.

Para quantificação de $H$. pylori, as peças cirúrgicas fixadas em formol a 3,8\% foram submetidas a cortes transversais de antro-piloro e corpo distal, incluidos em parafina e cortados a $6 \mathrm{~m}$ de espessura em micrótomo rotativo. Foram utilizadas as colorações de Warthin-Starry e de Giemsa modificada 9. Para determinar a presença de $H$. pylori, os cortes corados em Giemsa modificado, não montados, foram estudados em campo de $1000 \mathrm{X}$ em óleo de imersão. Para quantificação da bactéria, os cortes corados e montados pela técnica de Warthin-Starry foram estudados em campo óptico com aumento de $400 \mathrm{X}$. Foram analisadas mais de 100 glândulas antrais por peça e qualificadas como 0 (ausência de H. pylori), 1 (1 a 10 bacilos presentes na luz da glândula), 2 (11 a 20 bacilos na luz da glândula) e assim sucessivamente. Para quantificação de $H$. pylori, foi atribuído que as glândulas qualificadas como 1 possuíam 10 bacilos, as qualificadas como 2 possuíam 20 bacilos e assim sucessivamente. Com estes dados estabeleceu-se a relação entre o número absoluto de glândulas com $H$. pylori e sem $H$. pylori, e calculado o percentual de glândulas comprometidas em relação ao total de glândulas estudadas.

A análise de quantificação de $H$. pylori no Grupo II foi realizada no 1/3 distal do estômago ressecado e no coto gástrico após a morte dos animais. $\mathrm{Na}$ primeira objetivou-se detectar a presença de bactérias por glândula no terceiro mês de vida. Na segunda, o intuito foi comparar os dois grupos de animais com um ano de vida.

Os animais tinham características de uma amostra simples ao acaso, isto é, eram semelhantes em raça, sexo, peso e idade, e foram escolhidos ao acaso para os dois braços do estudo. Optou-se 
pela mediana como medida de tendência central, por representar um valor que contém $50 \%$ dos dados acima e abaixo deste valor, e especialmente útil quando os números são dispersos e a amostragem reduzida. $\mathrm{O}$ teste não paramétrico de Wilcoxon foi escolhido porque os dados não apresentavam distribuição normal. Neste teste procedeu-se à comparação dos valores observados nos dois grupos, com nível de significância positivo se abaixo de 0,05 .

\section{RESULTADOS}

Não houve mortalidade operatória. Dois animais do Grupo II morreram no quarto e sétimo meses de pós-operatório e foram excluídos da análise. A avaliação do $1 / 3$ distal do estômago ressecado dos animais do Grupo II revelou, em todos eles, a presença de $H$. pylori na luz glandular. A quantificação, no entanto, só mostrou glândulas do tipo 1 e 2, isto é, com até 10 ou 20 bacilos. Os resultados encontramse no Tabela 1.

A análise macroscópica dos estômagos retirados após a morte dos animais, não mostrou evidências de lesões sugestivas de neoplasias. No Grupo I, a microscopia não evidenciou hiperplasia foveolar ou displasia em nenhuma das peças. No Grupo II, as al- terações epiteliais encontradas variaram do aumento do número de mitoses à displasias de diferentes graus, e até adenocarcinoma invasor. Com exceção de um único caso (n 6), todos os animais desenvolveram alterações histopatológicas relacionadas à neoplasia (Tabela 2).

A análise da quantificação de H. pylori mostrou que havia um percentual aumentado de glândulas comprometidas por esta bactéria no Grupo II (mediana do Grupo I de 18,21\% versus mediana do Grupo II de 39,96\%). Avaliada esta diferença estatisticamente pelo teste não paramétrico de Wilcoxon, obteve-se o valor $\mathrm{W}=103,5$ e $\mathrm{P}=0,01$, mostrando-se, portanto, significativa. Os dois animais que desenvolveram adenocarcinoma invasor ( 2 e n 5) tiveram um percentual de comprometimento glandular superior à mediana de seu grupo $(47,68 \%$ e $40,74 \%$ respectivamente, versus $39,96 \%$ ) (Tabela 2).

\section{DISCUSSÃO}

A primeira referência à possível infecção gástrica por $H$. pylori foi feita por Bottcher ${ }^{10}$, em 1874, que relatou a presença de microrganismos espiralados no estômago de mamíferos. Em 1906, Kreinitz ${ }^{11}$ identificou estas bactérias na espécie humana. Marshall ${ }^{12}$, em 1982, isolou o germe através

Tabela 1 - Quantificação das lâminas com coloração de Warthin-Starry, do antro gástrico dos animais do Grupo 2.

\begin{tabular}{ccccc}
\hline ANIMAL & N. TIPO 1 * & N. TIPO 2** & $\begin{array}{c}\text { GLAND. COMP. / } \\
\text { GLAND. LIVRES*** }\end{array}$ & $\begin{array}{c}\text { \% DE COMP. } \\
\text { TOTAL**** }\end{array}$ \\
\hline 1 & 20 & 0 & $20 / 91$ & 18,01 \\
2 & 14 & 1 & $15 / 91$ & 14,15 \\
3 & 20 & 0 & $20 / 94$ & 17,54 \\
4 & 21 & 1 & $22 / 103$ & 16,93 \\
5 & 24 & 4 & $28 / 102$ & 21,53 \\
6 & 17 & 0 & $17 / 98$ & 14,78 \\
7 & 14 & 0 & $14 / 103$ & 11,96 \\
8 & 13 & 1 & $14 / 95$ & 12,84 \\
9 & 21 & 0 & $21 / 102$ & 17,07 \\
10 & 26 & 2 & $28 / 97$ & 22,40 \\
\hline
\end{tabular}

* Número de glândulas com $\mathrm{H}$. Pylori quantificadas como 1.

** Número de glândulas com H. Pylori quantificadas como 2.

*** Número absoluto de glândulas comprometidas e glândulas não comprometidas.

**** Percentual de glândulas com $\mathrm{H}$. pylori em relação ao total de glândulas estudadas. 
Tabela 2 - Achados histopatológicos e concentração de H. pylori, após nove meses de pós-operatório.

\begin{tabular}{cccc}
\hline GRUPO & NÚMERO & HISTOPATOLOGIA & $\begin{array}{c}\text { \% DE GLAND } \\
\text { COMP./ TOTAL* }\end{array}$ \\
\hline I & 1 & NORMAL & 0 \\
I & 2 & NORMAL & 38 \\
I & 3 & NORMAL & 23,5 \\
I & 4 & NORMAL & 10,71 \\
I & 5 & NORMAL & 21,84 \\
I & 6 & NORMAL & 11,2 \\
I & 7 & NORMAL & 10,94 \\
I & 8 & NORMAL & 50 \\
I & 9 & NORMAL & 35,57 \\
I & 10 & NORMAL & 14,59 \\
\hline II & 1 & Aumento do número de mitoses; & 39,18 \\
& & displasia leve; hiperplasia foveolar leve & \\
\hline II & 2 & Adenocarcinoma; displasia & 47,68 \\
& & moderada/intensa; aumento do & 50 \\
\hline II & 3 & número de mitoses & 17,35 \\
\hline II & 4 & Displasia leve; hiperplasia foveolar leve & 40,74 \\
\hline II & 5 & displasia leve & 35,44 \\
\hline II & 6 & displasia moderada & 38,67 \\
\hline II & 7 & Adenocarcinoma; displasia moderada & 61,58 \\
\hline II & 8 & NORMAL & \\
\hline
\end{tabular}

* Percentual de glândulas positivas para $\mathrm{H}$. pylori / total de glândulas.

de cultura de biópsia gástrica endoscópica, em meio para Campylobacter. Assim, a bactéria recebeu inicialmente o nome de Campylobacter pylori .

Em 1983, Warren ${ }^{13}$ demonstrou que a maioria dos pacientes portadores de úlcera péptica e gastrites tinham infecção por Campylobacter pylori. Goodwin et al ${ }^{14}$, em 1989, demonstraram que esta bactéria tem o 16S RNA ribossomal diferente do gênero Campylobacter, e caracterizaram um novo gênero, o Helicobacter. Trata-se de um bastonete Gramnegativo curvo ou espiralado, microaerófilo, equipado com quatro a seis flagelos em uma das extremidades ${ }^{15}$. É capaz de colonizar apenas o epitélio gástrico, podendo ser encontrado também em mucosa gástrica ectópica, como no divertículo de Meckel e no esôfago de Barrett.

A infecção por H. pylori induz uma resposta imunológica caracterizada pela formação de anticorpos do tipo IgG. Seus principais aspectos patogênicos são $0^{16}$ :

1. Produção de urease, que permite a degradação da uréia, alcalinizando o meio e favorecendo seu crescimento, além de efeito lesivo direto sobre a mucosa.

2. Produção de adesinas, substâncias que mantém a bactéria aderida à superfície epitelial e capazes de levar à liberação de fatores nocivos diretamente na membrana celular.

3. Degradação de nitritos e nitratos em Ncompostos, lesivos ao epitélio.

4. Estimulação de respostas inflamatórias no epitélio, com formação de radicais livres e outros produtos da resposta inflamatória que também são lesivos ao epitélio gástrico

O teste histológico, usado em nosso trabalho, deve ser a opção quando se realiza endoscopia. A 
bactéria é mais abundante no antro e, por isto, preconiza-se realizar as biopsias $1 \mathrm{a} 2 \mathrm{~cm}$ acima do anel pilórico. A análise histopatológica pode identificar a bactéria, mas sua quantificação tem se mostrado de difícil reprodução entre vários examinadores pois é feita de forma subjetiva ${ }^{17}$.

Dentre as doenças gastro-duodenais produzidas por $H$. pylori , além da úlcera duodenal e da úlcera gástrica, chama a atenção a gastrite crônica, que pode evoluir com metaplasia intestinal nos casos de agressão continuada ${ }^{18-23}$. A ocorrência de displasia também pode ser observada em alguns casos, o que conduziu ao estabelecimento da relação $H$. pylori / adenocarcinoma, e levou a World Health Organization (WHO), em 1994, a considerar o H. pylori como fator carcinogenético ${ }^{16,24-26}$. Diferentes estudos epidemiológicos com testes sorológicos, estabeleceram forte correlação entre a incidência de adenocarcinoma e a positividade sorológica para $H$. pylori ${ }^{24,25,27}$. A sequiência de eventos para indução de neoplasia seria: gastrite crônica atrófica, displasia, câncer. O H. pylori é sabidamente indutor dos dois primeiros passos desta seqüência.

$\mathrm{Na}$ teoria de carcinogênese proposta por Parsonnet $^{28}$, a infecção por $H$. pylori atuaria de duas formas: induzindo proliferação epitelial com possibilidade de erros mitóticos e produzindo fatores mutagênicos relacionados à inflamação. Neste ponto comum, atuariam os carcinógenos dietéticos como agravantes e os anti-oxidantes dietéticos e mecanismos celulares de reparo do ADN como fatores protetores. $\mathrm{O}$ câncer gástrico teria origem a partir de um desequilíbrio a favor das células mutantes.

No que se refere à correlação entre gastrectomia e câncer do coto gástrico (CCG), foi demonstrada uma incidência de $1 \%$ no período de 28 anos após a cirurgia, com risco igual ao da população geral aos 15 anos e aumento crescente a partir dos 20 anos de gastrectomia ${ }^{29-31}$. Quanto à sua carcinogênese, o CCG é atribuído ao refluxo êntero-gástrico, que provocaria alterações na mucosa do tipo gastrite , metaplasia intestinal e hiperplasia foveolar, que, alterando a cinética celular poderia levar à displasia e/ou neoplasia epitelial ${ }^{30,32-34}$.

A patogênese da gastrite alcalina de refluxo está relacionada ao efeito detergente da bile sobre o muco gástrico, à alterações da secreção de bicarbonato pelas células gástricas e à liberação de diferentes substâncias, como prostaglandinas, que possibilitariam a retrodifusão de íons hidrogênio com consequente gastrite ${ }^{35,36}$. Mais recentemente, chamou-se a atenção para a relação entre gastrite alcalina e H.pylori ${ }^{37,38}$. Estudos em seres humanos mostraram que a flora bacteriana do estômago após gastrectomia produz maior quantidade de nitrosaminas, que estão, sabidamente relacionadas ao desenvolvimento de câncer gástrico ${ }^{33}$. Nagahata ${ }^{39}$, estudou o papel do $H$. pylori na etiologia da gastrite de refluxo em pacientes submetidos à gastrectomia. Demonstrou que $40 \%$ eram $H$. pylori positivos na biopsia e que, após o tratamento, houve melhora significativa dos sintomas e da avaliação histológica da gastrite. Ladas et al ${ }^{40}$ estudou 38 pacientes não operados, visando a determinar se a colonização antral por $H$. pylori e refluxo duodeno-gástrico seriam independentes ou estariam correlacionados. Identificou pacientes portadores de refluxo, através de cintilografia BrIDA-Tc, e de H.pylori, através de biopsia, e tratou clinicamente a infecção. Após seis meses realizou novo estudo e demonstrou alta taxa de desaparecimento do refluxo no grupo tratado que permaneceu H.pylori negativo. Outros autores encontraram resultados semelhantes estudando a correlação entre H.pylori e gastrite alcalina de refluxo pós-gastrectomia a BII ${ }^{41}$.

A etiopatogenia do câncer gástrico permanece indefinida. O papel do H.pylori, no entanto, vem despertando grande interesse. Considerada definitiva nos linfomas gástricos do tipo MALT ${ }^{42-44}$, esta correlação vem se tornando cada vez mais forte também quanto ao adenocarcinoma, particularmente do tipo intestinal de Lauren. Estudos epidemiológicos e de determinação de anticorpos para H.pylori, demonstraram forte paralelo entre a infecção por este agente e o desenvolvimento de câncer gástrico, com risco estimado de até seis vezes maior quando comparado a indivíduos não infectados ${ }^{28}$.

A carcinogênese experimental induzida pelo H.pylori pode estar relacionada à sua capacidade de produzir urease, enzima que forma amônia no estômago, gerando compostos nitrogenados ${ }^{45}$.

Estudos em que se utilizou o N-methyl-N'nitro-N-nitosoguanidina (MNNG) demonstraram que o H.pylori atua independentemente deste carcinógeno ${ }^{46}$.

A comparação experimental da correlação entre H.pylori e câncer gástrico é, portanto, de grande interesse, assim como, por conseguinte, a possibilidade de prevenção da neoplasia através da erradicação da bactéria. 
Divergências metodológicas na quantificação de H.pylori, porém, tem dificultado os estudos, já que em diferentes trabalhos científicos a infecção gástrica tem sido relatada de forma subjetiva como leve, moderada e intensa, o que dificulta a reprodução do método entre diferentes pesquisadores. Desta forma idealizou-se um modelo experimental de quantificação que pudesse ser expresso numericamente, no qual, a contagem de H.pylori é baseada no número de bactérias por glândula gástrica, o que permitiu não só uma quantificação em números absolutos, mas também a determinação do pércentual de glândulas infectadas sobre o total estudado.

A escolha dos dois parâmetros histopatológicos estudados, a hiperplasia foveolar e a displasia, baseou-se na cadeia de eventos ligados à tese da formação do adenocarcinoma gástrico induzido por H.pylori e por refluxo. A hiperplasia foveolar gástrica é considerada o mais confiável marcador histológico de refluxo êntero-gástrico no estômago operado e intacto ${ }^{37,45,47}$. Na hipótese de formação de adenocarcinoma gástrico induzido por H.pylori, admite-se a seqüência de gastrite crônica atrófica, metaplasia intestinal, displasia ${ }^{48}$. No caso da displasia, importante condição pré-maligna, optou-se pelos critérios de Ming ${ }^{8}$ para o seu diagnóstico e classificação, pela universalidade dos mesmos, aliados à sua fácil reprodução.

A relação entre gastrectomia e câncer do coto gástrico sempre foi considerada importante, com trabalhos demonstrando uma probabilidade duas a quatro vezes maior após vinte anos da ressecção gástrica, quando comparado à população em geral ${ }^{49}$. O estudo da microbiota do estômago operado revela aumento do número de bactérias capazes de produzir nitrosaminas ${ }^{33}$. Silva ${ }^{50}$ mostrou não só um aumento da população bacteriana do coto gástrico, como uma relação desta com o tipo de reconstrução. Animais com reconstrução em Y de Roux, ou seja, sem refluxo êntero-gástrico, tiveram índices menores de proliferação bacteriana. Trabalhos mais recentes demonstram proliferação de H.pylori no coto gástrico de pacientes submetidos a gastrectomia a BII e associam esta bactéria ao desenvolvimento de gastrite ${ }^{39-41}$.

A sequiência de eventos produzidos pelo refluxo êntero-gástrico e que culmina em gastrite atrófica parece ser semelhante aqueles produzidos pela infecção por H.pylori, embora não se possa descartar a possibilidade de ação conjunta entre estes dois fatores.

Nosso estudo confirmou os achados do trabalho pioneiro de Langhans ${ }^{51}$, que induziram câncer do coto gástrico em ratos sem a administração de carcinógenos. Embora nove meses possam parecer um curto período de tempo, equivalem a 15/20 anos em humanos, período de maior risco de desenvolvimento de câncer do coto gástrico.

Os resultados do nosso trabalho também enfatizam a relação entre refluxo êntero-gástrico e H. pylori e permitem supor que a intensidade da infecção gástrica associada ao refluxo possam estar ligadas ao desenvolvimento de câncer do coto gástrico de ratos. O grau de comprometimento glandular gástrico por H.pylori aumenta com o refluxo ênterogástrico e está associado a maior incidência de alterações displásicas e neoplásicas no epitélio do coto gástrico, em ratos submetidos a gastrectomia parcial com reconstrução a BII.

\begin{abstract}
Background: Gastric carcinogenesis is still under investigation. Our objective is to evaluate a possible relationship between $\mathrm{H}$.pylori and entero-gastric reflux in the genesis of epithelial neoplasias after Billroth II gastrectomy in rats. Methods: Twenty male Wistar rats were divided into two groups. Group I rats underwent laparotomy and group II had a Billroth II gastrectomy performed. After nine months, survivors were killed and their stomachs analysed for quantification of $\mathrm{H}$.pylori and for pre-neoplastic and neoplastic changes of the epithelium. Results: Group II animals had a significant higher percentage of colonization by H.pylori and their epithelium had more pathological changes that ranged from a higher number of mitotic cells to invasive carcinoma. Conclusion: The percentage of glandular involvement by H.pylori increases with entero-gastric reflux after partial gastrectomy and is associated to the development of gastric stump cancer in rats.
\end{abstract}

Key Words: Stomach neoplasms; Gastrectomy; Helicobacter pylori; Wistar, rats. 


\section{REFERÊNCIAS}

1. Brumini R - Câncer no Brasil: dados histopatológicos de 1976-1980.Campanha Nacional de Combate ao Câncer. Ministério da Saúde. Rio de Janeiro. 1982, p. 4-13.

2. INSTITUTO NACIONAL DE CÂNCER PRÓ-ONCO. Estimativa da incidência e mortalidade por câncer no Brasil. Rio de Janeiro, Ministério da Saúde, 1997.

3. Matsukura N, Kawachi T, Sasajima K, et al. - Induction of intestinal metaplasia in the stomachs of rats by $\mathrm{N}$ methyl-N'-nitro-N-nitrosoguanidine. J Natl Cancer Inst, 1978, 61(1): 141-144.

4. Sasajima K, Kawachi T, Matsukura N, et al. - Intestinal metaplasia and adenocarcinoma induced in the stomach of rats by N-propyl-N'-nitro-N-nitrosoguanidine. J Cancer Res Clin Oncol, 1979, 94(2): 201-206.

5. Mason RC - Duodenogastric reflux in rat gastric carcinoma. Br J Surg, 1978, 73(10): 801-803.

6. Correa P, Ruitz B - Campylobacter pylori and gastric cancer. In Rathbone BJ, Heatly RV. Campylobacter pylori and gastroduodenal disease. Oxford. Blackwell Scientific Publication,1989, pp. 139-145.

7. Rakic S, Bandovic J, Dunjic M, et al. - Gastric foveolar hyperplasia in patients with cancer of the intact stomach. Surg Laparosc Endosc, 1994, 4(3): 196-199.

8. Ming SC, Bajtai A, Correa P, et al. - Gastric dysplasia. Significance and pathologic criteria. Cancer, 1984, 54(9): 1794-1801.

9. Rocha GA, Queiroz DM, Mendes EN, et al. - Simple carbolfuchsin staining for showing $\mathrm{C}$ pylori and other spiral bacteria in gastric mucosa. J Clin Pathol, 1989,42(9): 1004-1005.

10. Bottcher G - Dorpater Med Z, 1974, 5: 148.

11. Krienitz W. Ueber das auftreten von spirochaetne verschiegener form im magen nhalt bei carcinima ventriculi. Deustsch Med Wochenschr, 1906, 32:872.

12. Marshall BJ, Warren JR - Unidentified curved bacilli in the stomach of patients with gastritis and peptic ulceration. Lancet, 1984, 1(8390): 1311-1315

13. Warren, JR - Unidentified curved bacilli on gastric epithelium in active chronic gastritis (letter). Lancet, 1983, 1(8336): 1273-5.

14. Goodwin CS, Armstrong JA, Chilvers T, et al. Transfer of Campylobacter pylori and Campylobacter mustelae to Helicobacter gen. nov. as Helicobacter pylori comb. nov. and Helicobacter mustelae comb. nov., respectively. Int J Syst Bacteriol, 1989, 39(4): 397-405.

15. Parsonnet J, Blaser MJ, Perez-Perez GI, et al. Symptoms and risk factors of Helicobacter pylori infection in a cohort of epidemiologists. Gastroenterology, 1992, 102(1): 41-46.

16. Calam J - Helicobacter pylori. London. Chapman and Hall Medical, 1996.
17. Atherton JC, Spiller RC - The urea breath test for Helicobacter pylori. Gut, 1994, 35(6): 723-725.

18. Armstrong D - Helicobacter pylori infection and dyspepsia. Scand J Gastroenterol, 1996, 215(Suppl): 38-47.

19. Dixon MF, Genta RM, Yardley JH, et al. - Classification and grading of gastritis. The updated Sydney System. International Workshop on the Histopathology of Gastritis. Houston 1994. Am J Surg Pathol, 20(10): 1996, 1161-1181.

20. Pinheiro JO, Mattos AA - Etiopatogenia da gastropatia da hipertensão portal: o papel do Helicobacter pylori. GED Gastroenterol Endosc Dig, 1997,16(4): 111-118.

21. Labenz J, Tillenburg B, Peitz U, et al. - Long-term consequences of Helicobacter pylori eradication: clinical aspects. Scand J Gastroenterol, 1996, 215(Suppl):111-115.

22. Kuipers EJ, Appelmelk BJ - Helicobacter pylori and atrophic gastritis. Biomed Pharmacother, 1997, 51(4):150-155.

23. Kuipers EJ, Klinkenberg-Knol EC, VandenbrouckeGrauls CM, et al. - Role of Helicobacter pylori in the pathogenesis of atrophic gastritis. Scand J Gastroenterol, 1997, 223(Suppl): 28-34.

24. Asaka M - [Helicobacter pylori and malignant stomach neoplasms] Nippon Naika Gakkai Zasshi, 1996, 85(5): 767-771.

25. Gonzalez CA, Sanz JM, Agudo A - Factores de riesgo del cancer gastrico. Gastroenterol Hepatol, 1997, 20(5): 239-247.

26. Matsui T, Matsukawa Y, Sakai T, et al. - Ammonia inhibits proliferation and cell cycle progression at Sphase in human gastric cells. Dig Dis Sci, 1997, 42(7): 1394-1399.

27. Watanabe Y, Kurata JH, Mizuno S, et al. - Helicobacter pylori infection and gastric cancer. A nested casecontrol study in a rural area of Japan. Dig Dis Sci , 1997, 42(7): 1383-1387.

28. Parsonnet $\mathbf{J}$ - Helicobacter pylori and gastric cancer. Gastroenterol Clin North Am, 1993, 22(1): 89-104.

29. Toftgaard C - Gastric cancer after peptic ulcer surgery. A historic prospective cohort investigation. Ann Surg, 1989, 210(2): 159-164.

30. Cheli R, Santi L, Ciancamerla G, et al. - A clinical and statistical follow-up study of atrophic gastritis. Am J Dig Dis, 1973, 18(12): 1061-1065.

31. Nicholls JC-Carcinoma of the stomach following partial gastrectomy for benign gastroduodenal lesions. $\mathrm{Br} \mathrm{J}$ Surg, 1974, 61(3): 244-249.

32. Morgenstern L - Vagotomy, gastroenterostomy and experimental gastic cancer. Arch Surg, 1968, 96(6): 920-931.

33. Muscroft TJ, Deane SA, Youngs D, et al. - The microflora of the postoperative stomach. Br J Surg, 1981, 68(8): 560-564. 
34. Offerhaus GJ, van de Stadt J, Huibregtse K, et al. - The mucosa of the gastric remnant harboring malignancy. Histologic findings in the biopsy specimens of 504 asymptomatic patients 15 to 46 years after partial gastrectomy with emphasis on nonmalignant lesions. Cancer, 1989, 64(3): 698-703.

35. Vieira OM - Gastrite alcalina de refluxo. (Estudo experimental sobre a etiopatogenia). Dissertação. Rio de Janeiro. Universidade Federal do Rio de Janeiro, 1977.

36. Vieira OM -Tratamento da gastrite alcalina de refluxo. Dissertação. Rio de Janeiro. Universidade Federal do Rio de Janeiro, 1987.

37. Bechi P - Helicobacter pylori and duodenogastric reflux. Gut, 1996, 39(5): 773-774.

38. Caldwell MT, McDermott M, Jazrawi S, et al. Helicobacter pylori infection increases following cholecystectomy. Ir J Med Sci,1995,164(1): 52-55.

39. Nagahata Y, Kawakita N, Azumi Y, et al. - Etiological involvement of Helicobacter pylori in "reflux" gastritis after gastrectomy. Am J Gastroenterol, 1996, 91(10): 2130-2134.

40. Ladas SD, Katsogridakis J, Malamou H, et al. Helicobacter pylori may induce bile reflux: link between $\mathrm{H}$ pylori and bile induced injury to gastric epithelium. Gut, 1996, 38 (1): 15-18.

41. Kawakita N, Nagahata Y, Azumi Y, et al. - [Residual gastritis after gastrectomy and Helicobacter pyloriits clinic significance]. Nippon Shokakibyo Gakkai Zasshi, 1995, 92(5): 862-869.

42. Neff G, Van Ness MM, Dies DF - Helicobacter pylori: current concepts. J La State Med Soc, 1996, 148(8): 364-366.

43. Walt RP - Regression of MALT lymphoma and treatment for Helicobacter pylori. Lancet, 1996, 348(9033): 1041-1042.

44. Williams $\mathrm{CN}$ - Helicobacter pylori, gastric cancer and gastric MALT lymphoma.Can J Gastroenterol, 1996,10(6):359-360.
45. Tsujii M, Kawano S, Tsuji S, et al. - Ammonia: a possible promotor in Helicobacter pylori-related gastric carcinogenesis. Cancer Lett, 1992, 65(1): 15-18.

46. Kawaura A, Yamamoto I, Tanida N, et al. - Helicobacter pylori is not a co-carcinogen in N-methyl-N'-nitro-Nnitrosoguanidine-induced rat gastric carcinogenesis. Tokushima J Exp Med, 1991, 38(3-4): 71-75.

47. Parsonnet J, Friedman GD, Vandersteen DP, et al. Helicobacter pylori infection and the risk of gastric carcinoma. NEngl J Med, 1991, 325(16): 1127-1131.

48. Sipponen P - Helicobacter pylori and gastric premalignant conditions. Acta Gastroenterol Belg, 1991, 54(5-6): 340-344.

49. Caygill CP, Hill MJ, Kirkham JS, et al. - Mortality from gastric cancer following gastric surgery for peptic ulcer. Lancet, 1986, 1(8487): 929-931.

50. Silva, LF - Estudo da proliferação bacteriana no trato gastrointestinal superior pós vagotomia e ressecção gástrica. Dissertação (Doutorado). Rio de Janeiro. Universidade Federal do Rio de Janeiro, 1980.

51. Langhans P, Heger RA, Hohenstein J, et al. - Operationsequel carcinoma of the Stomach. Experimental studies of surgical techniques with or without resection. World J Surg, 1981, 5(4): 595-605.

Agradecimentos: Ao Prof. Pedro de Carvalho, professor de estatística do INCa que procedeu à análise dos dados.

Endereço para correspondência:

Eduardo Linhares Riello de Mello Instituto Nacional do Câncer

Praça da Cruz Vermelha, 23

Centro - Rio de Janeiro - RJ

E-mail: riello@openlink.com.br 